\title{
Turbulent velocity profiles in a tilted heat pipe
}

\author{
J. Salort, X. Riedinger, ${ }^{*}$ E. Rusaouen, J.-C. Tisserand, F. Seychelles, B. Castaing, and \\ F. Chillà ${ }^{\dagger}$ \\ Laboratoire de Physique de l'Ecole Normale Supérieure de Lyon \\ UMR5672 - 46 allée d'Italie, 69364 Lyon Cedex 07, France
}

(Dated: September 13, 2013)

\begin{abstract}
In this paper, we analyze the mean velocity profile and the Reynolds shear stress in a turbulent, inclined, heat pipe. We show that the simplest version of a mixing length model is unable to reproduce the evolution of the velocity profile shape with the inclination angle $\psi$. An improvement of this model, taking into account some buoyancy effects, gives nice qualitative agreement with the observations. The agreement implies surprisingly a low value for the gradient Richardson number $R i_{c}$ above which the flow is laminar. The comparison with previous works shows however good agreement when some care is taken in the evaluation of the Richardson number $R i$.
\end{abstract}

PACS numbers: 47.2747 .55

\section{INTRODUCTION}

In a recent paper [1], we carefully analyzed the thermal behavior of a square heat pipe, depending on its inclination angle and the applied heat flux. From the four regimes we could distinguish, two of them, we called Hard and Soft Turbulence, suggested an interesting interplay between turbulence and stratification. The goal of the present paper is to compare the mean flow and mean Reynolds stress, obtained from Particle Image Velocimetry (PIV) measurements, with the conclusions of a mixing length model. With this model, we aim at clarifying the relation between the mean flow and Reynolds stresses.

The Prandtl mixing length model $[2,3]$ is the first attempt to close the Reynolds averaged equations of turbulent flows. Despite the development of more elaborate models, this Prandtl mixing length model (ML) keeps some advantages, in terms of physical interpretation, and possibility of analytical solutions. Moreover, the development of Particle Image Velocimetry (PIV), for the analysis of experimental flows, and of Direct Numerical Simulations (DNS) of high resolution, allow to directly test the founding hypotheses of ML.

There has been other similar attempts [4-7] to get insight in the physics of convection through a mixing length model. Indeed, free convection is responsible of most of natural flows. While its driving mechanism is conceptually simple, it can result in very complex flows, due to the interplay between mixing, buoyancy and transport for the active scalar. There is a need to clarify the relations between the large scale flow and transport, both scalar and momentum transport (stresses).

Heat pipes, or gravital flows in vertical or inclined

\footnotetext{
*Also at College of Engineering, Mathematics and Physical Sciences, University of Exeter, North Park Road, Exeter, UK, EX4 $4 \mathrm{QF}$.

$\dagger$ Electronic address: Francesca.Chilla@ens-lyon.fr
}

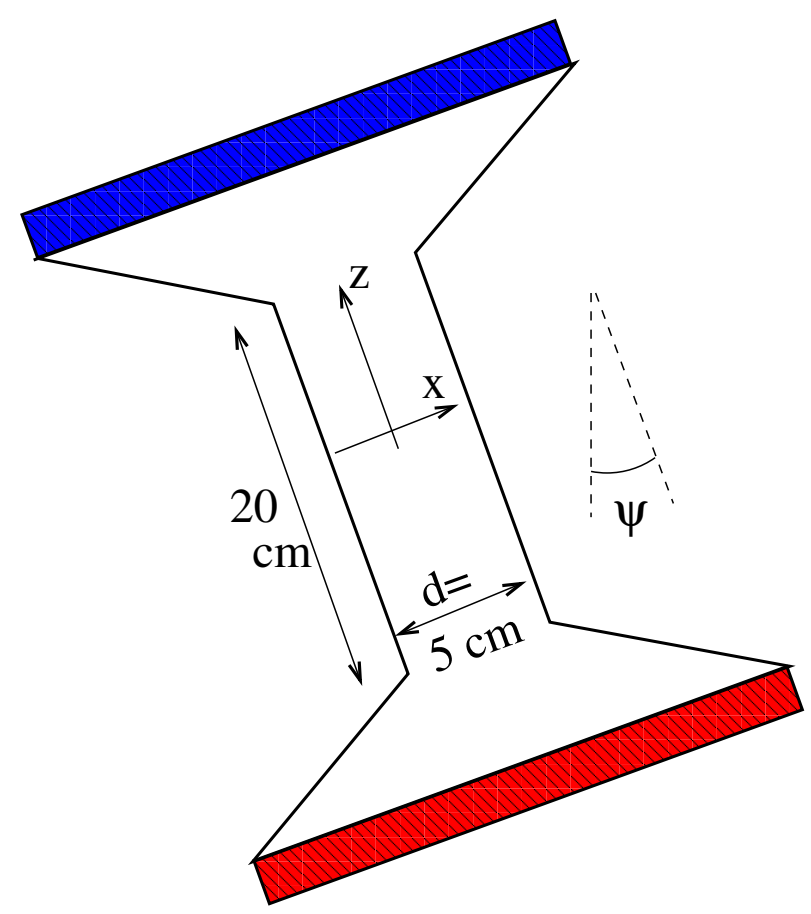

FIG. 1: Sketch of the experimental cell with the definition of coordinates.

tubes allow to study these relations with simple, homogeneous large scale flows. In the past decade, several works have aimed at characterizing the different flow regimes in such a geometry [1, 8-14]. This paper is one of a series of five papers, each concentrating on one aspect of the same system, schematized in figure 1 and described in section II. In the first one [9], we focused on the vertical case. In our previous paper [1], we concentrated on the thermal aspect with various inclination angles $\psi$, which allowed to evidence several regimes, from turbulent to laminar. Two papers are in preparation, one interested in the Laminar regime, and its destabilization through intermittent bursts, the other detailling all the statistical 
aspects of the turbulent regimes, like structure functions and probability density functions.

The present paper is thus devoted to clarify the phenomenological relations between the mean shear flow, the mean temperature profile, the Reynolds stresses and the transverse heat flux in the turbulent regimes, and to interpret them through a Mixing Length model. The paper is organized as follows. In section II, we recall the main characteristics of our experimental set-up. In section III, we summarize the experimental results obtained in the two turbulent regimes. In section IV, we determine the Reynolds equations governing this turbulent flow. In section $\mathrm{V}$ we discuss the consequences of the mixing length $[2,3]$ closure for these equations (equivalent to the Smagorinsky [15] sub-grid model). We show that its simplest version cannot take into account the evolution of the velocity profile with the inclination angle $\psi$ experimentally observed. In section VI, we examine a modified version of this closure, proposed in $[16,17]$ for taking into account the stratification due to the gravity. Finally, in section VII, we compare with experimental results, before to conclude, section VIII.

\section{EXPERIMENTAL SET-UP}

All the details about the experimental set-up can be found in references $[1,9]$ where we refer. We simply recall here the most important points, relative to the subject of this paper. The definition of coordinates is shown in the sketch of the cell, figure 1. This cell, filled with water, consists in two conical chambers, of height $10 \mathrm{~cm}$, based by circular copper plates, $20 \mathrm{~cm}$ in diameter. The two chambers are connected through a square channel $d \times d=5 \times 5 \mathrm{~cm}^{2}$ of inner dimensions, $20 \mathrm{~cm}$ long. The hot plate is Joule heated by a spiral resistor. The cold plate is temperature regulated by a water bath.

The velocity field is measured by Particle Image Velocimetry (PIV) technique. We use a one watt green continuous Melles Griot laser (DPSS-Laser System, 532nm, Melles Griot), and cylindrical lens for creating a laser sheet. Our water is sown with hollow glass particles (Sphericel 110P8, LaVision, GmbH), 10 micrometers in average diameter. Both the recording and the batch processing are done with the Davis Lavision software.

We worked by series of 3 images, with $40 \mathrm{~ms}$ between these images ( 2 velocity fields for each series). The series are separated by $10 \mathrm{~s}$. A record consists in 1000 series (which corresponds to 2 hours and 50 minutes). All the records are made at an average temperature of $25^{\circ} \mathrm{C}$, close to the room temperature, to minimize possible heat leaks. The Prandtl number of water at this temperature is $\operatorname{Pr}=\nu / \kappa=6$, where $\nu$ is the kinematic viscosity, and $\kappa$ the heat diffusivity.

In the vertical case, treated in previous papers $[8,9]$, the flow underwent reversals, oscillating between two main modes, the hot rising fluid being either at the right or at the left side of the channel. In this case, these two

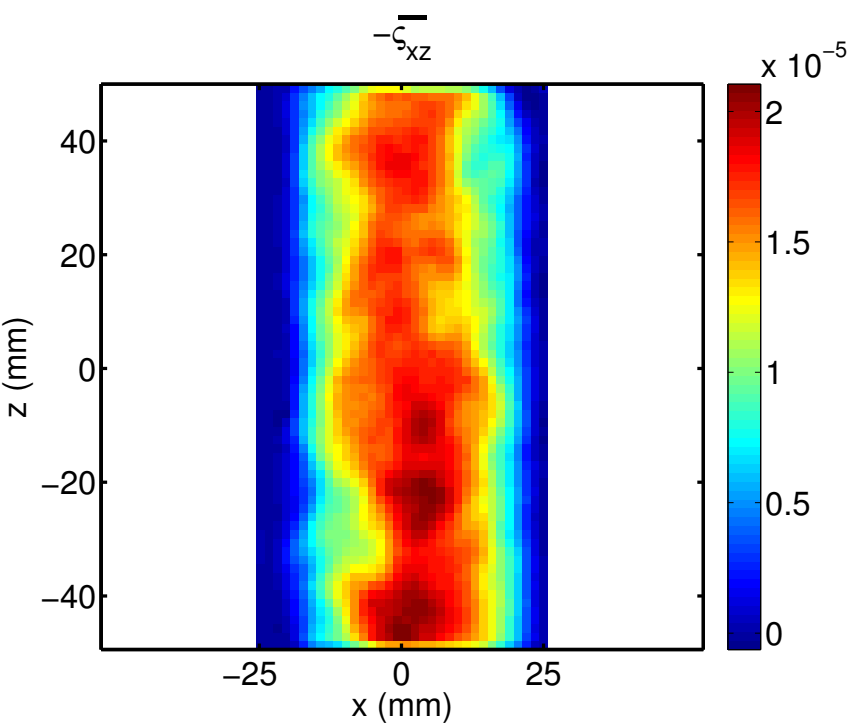

FIG. 2: Map of the time averaged transverse Reynolds stress $\varsigma_{x z}=\left\langle v_{z}^{\prime} v_{x}^{\prime}\right\rangle\left(\right.$ in $\left.\mathrm{m}^{2} \mathrm{~s}^{-2}\right)$. The inclination angle is $\psi=10^{\circ}$, and the applied heat power is $77 \mathrm{~W}\left(U_{t} \simeq 1 \mathrm{~cm} / \mathrm{s}\right)$

modes are equivalent, being symmetric versus the vertical $z y$ plane. It required us to artificially rectify the flow, applying the above symmetry to the data when necessary. Without this rectification, the mean flow and the transverse Reynolds stress would appear as zero everywhere [14]. These reversals disappear rapidly when $\psi$ is increased. With $\psi=5^{\circ}$, the flow is $90 \%$ of the time in the most frequent mood, the hot fluid rising along the upper face, and the cold one diving along the lower. However, in this $\psi=5^{\circ}$ case, we found necessary to exclude from the statistics the velocity fields where a reversal occured. As in the vertical case, once averaged in time, the various statistical quantities are reasonably independent of $z$ (see for example the transverse Reynolds stress shown in figure 2). We thus increase the statistics by averaging on $z$ in the most central $10 \mathrm{~cm}$ of the channel: $\langle$.$\rangle means$ averaged on time and $z$, while - means averaged only on time. We define the fluctuations as $v_{z}^{\prime}=v_{z}-\left\langle v_{z}\right\rangle$. The averaged transverse velocity $\left\langle v_{x}\right\rangle$ is negligible, thus $v_{x}^{\prime}=v_{x}$. For the purpose of this paper, we further increased artificially the statistics by symmetrizing the profiles. Indeed, the longitudinal velocity profile, its even $x$-derivatives, and the odd $x$-derivatives of the Reynolds stresses are anti-symmetric through the symmetry $x \rightarrow-x$. In the same way, the Reynolds stresses and their even $x$-derivatives, and the odd $x$-derivatives of the longitudinal velocity profile, are invariant in the same symmetry. 


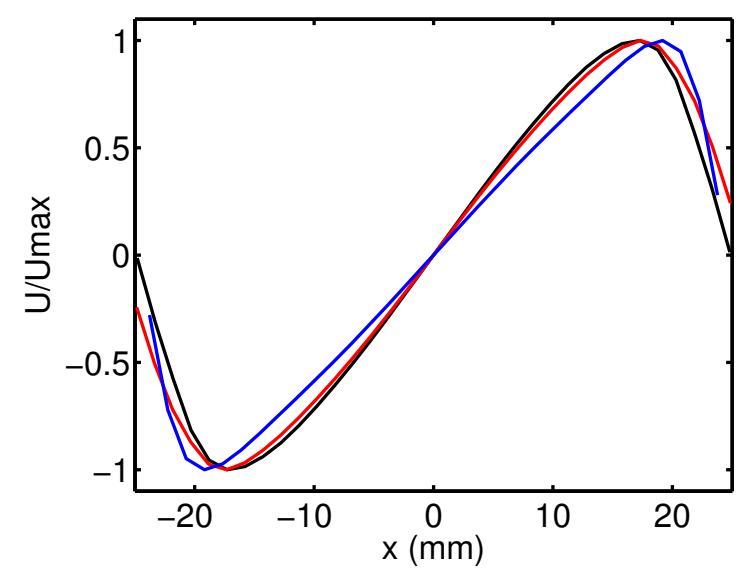

FIG. 3: Symmetrized velocity profiles normalized to their maximum. From the largest to the smallest middle slope: $\psi=5^{\circ}$ (black), $\psi=10^{\circ}$ (red on line), $\psi=20^{\circ}$ (blue on line). The applied heat power is $77 \mathrm{~W}\left(U_{t} \simeq 1 \mathrm{~cm} / \mathrm{s}\right)$

\section{SUMMARY OF EXPERIMENTAL OBSERVATIONS}

In our previous paper [1], the Soft and Hard turbulent regimes distinguished themselves through the heat transport along the tube. They present different power law relations between the longitudinal temperature gradient, we call $\beta$, and the longitudinal heat flux $c_{p} Q_{z}$, where $c_{p}$ the isobaric heat capacity per unit volume (for simplicity, from now on, we shall speak of longitudinal heat flux for $\left.Q_{z}\right)$. For the Hard Turbulence regime, which is found at small inclination angles $\psi$, and high heat fluxes $Q_{z}$, we find $\beta \propto Q_{z}^{2 / 3}$. For larger $\psi$, or smaller heat fluxes, we find the Soft Turbulence, where $\beta \propto Q_{z}^{1 / 3}$. The interpretation is that Hard Turbulence exhibits a range of inertial scales between the correlation length and the dissipative scale (see [1]). In the Soft Turbulence, the Reynolds number is not sufficient for an inertial range to develop. For inclination angles $\psi>20^{\circ}$, Riedinger et al. [1] could only observe intermittent or laminar flows. It is why we limit ourselves to $\psi \leq 20^{\circ}$.

In previous papers $[1,9]$, a heat power dependent characteristic velocity $U_{t}$ was defined:

$$
U_{t}=\left(-g_{z} \alpha Q_{z} d\right)^{1 / 3} \quad, \quad Q_{z}=\frac{P}{c_{p} d^{2}},
$$

where $d=5 \mathrm{~cm}$ is the size of the channel, $\vec{g}$ is the gravity acceleration, $\alpha$ is the isobaric thermal expansion, and $P$ is the power applied to the hot plate. Once normalized to this velocity, the longitudinal velocity profile is independent of the heat power applied, both in the Hard and the Soft Turbulence. However, both the amplitude and the shape of this normalized profile depend on the inclination angle $\psi$.

The change of shape of the mean longitudinal velocity

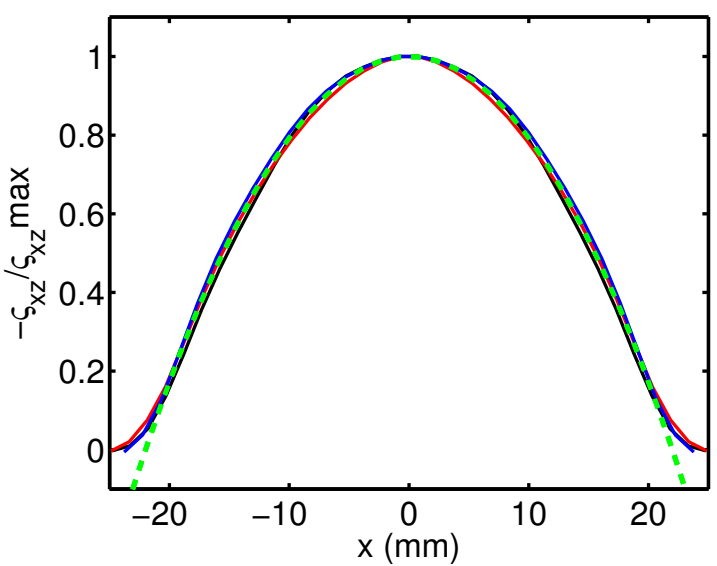

FIG. 4: Transverse Reynolds stresses, normalized to their maximum absolute value, for the same angles and the same applied power than in figure 3 . We also show the quadratic fit $1-4 x^{2} / d_{\text {eff }}^{2}$, which allows to determine $d_{\text {eff } f}$.

profiles (figure 3 ):

$$
U_{z}(x)=\left\langle v_{z}\right\rangle \quad,
$$

is one of the most striking effects of the tilting of the channel.

In the vertical case, treated in previous papers $[8,9]$, the profile was found close to a sine function. In the inclined case, the profile is more linear. To stress this difference, we normalize in figure 3 the profile to its maximum value. The various curves correspond to $\psi=5^{\circ}, 10^{\circ}$, and $20^{\circ}$. The evolution is clear, the profiles being more and more linear, when going from $\psi=5^{\circ}$ to $\psi=20^{\circ}$.

On the other hand, the profiles of the Reynolds shear stresses show no qualitative difference between them. In figure 4 , we present the shear turbulent stress $\varsigma_{x z}=$ $\left\langle v_{x}^{\prime} v_{z}^{\prime}\right\rangle$, normalized to its maximum. The curves correspond to the same angles $\psi=5^{\circ}, 10^{\circ}$, and $20^{\circ}$, with the same color code as figure 3 . It can be pointed out, as in the vertical case $[8,9]$, that the longitudinal $(z)$ fluctuations are more uniform than the transverse $(x)$ ones. This will be discussed in a next paper. All the presented curves have been realized with an applied power of $P=77 \mathrm{~W}$, corresponding to $U_{t} \simeq 1 \mathrm{~cm} / \mathrm{s}\left(\cos \left(20^{\circ}\right) \simeq 0.94\right.$ is close to 1).

Also shown in figure 4 is the fit of the normalized stress $\left(\psi=20^{\circ}, P=77 \mathrm{~W}\right)$ by a quadratic function:

$$
1-\frac{4 x^{2}}{d_{e f f}^{2}}
$$

Such a fit allows to define $d_{\text {eff }}$ for each angle and power. We can see that $d_{\text {eff }}$ is the same in all the three presented cases, and, in general, remains close to $44 \mathrm{~mm}$.

To be explicit, the slope of the velocity profile $U_{z}(x)=$ 
$\left\langle v_{z}\right\rangle$ at the origin scales with $U_{t}$ :

$$
\partial_{x} U_{z}(0)=\frac{2}{d} \mathcal{C}_{v} U_{t}
$$

where $\mathcal{C}_{v}$ only depends on the inclination angle $\psi$. The shape of the velocity profile also depends on $\psi$, evoluting from a sine shape at small $\psi$ to a linear shape at larger $\psi$. The shear stress profile has a parabolic shape:

$$
\varsigma_{x z} \simeq \mathcal{C}_{\sigma} U_{t}^{2}\left(1-\frac{4 x^{2}}{d_{\text {eff }}^{2}}\right),
$$

where, again $\mathcal{C}_{\sigma}$ only depends on $\psi$. The coefficient $\mathcal{C}_{\sigma}$ and the length $d_{\text {eff }}$ are determined by fiting the experimental Reynolds stress profile with this parabolic profile, and $x= \pm d_{\text {eff }} / 2$ can be interpreted as the points where the Reynolds shear stress vanishes.

\section{REYNOLDS EQUATIONS}

We shall interpret our observations within a two dimensional model, assuming invariance in the $y$ direction, perpendicular to $x$ and $z$. Taking into account the incompressibility, within the Boussinesq approximation:

$$
\begin{gathered}
\partial_{t} v_{x}+\partial_{z}\left(v_{x} v_{z}\right)=-\partial_{x}\left(\frac{p}{\rho}+v_{x}^{2}\right)-g_{x} \alpha \theta+\nu \Delta v_{x} \\
\partial_{t} v_{z}+\partial_{x}\left(v_{x} v_{z}\right)=-\partial_{z}\left(\frac{p}{\rho}+v_{z}^{2}\right)-g_{z} \alpha \theta+\nu \Delta v_{z}
\end{gathered}
$$

$\rho$ is the density, $p$ is the pressure, corrected from the hydrostatic one at the average density, and $\alpha$ the isobaric thermal expansion coefficient of our fluid. We note $\theta$ the temperature, with the origin such that the average temperature is zero for $x=z=0$.

These equations 6 and 7 are invariant within the simultaneous transformations $x \rightarrow-x, z \rightarrow-z, \theta \rightarrow-\theta$, and $\vec{g} \rightarrow-\vec{g}$. The time average $\bar{\theta}$ of $\theta$ is $z$ and $x$ dependent: $\bar{\theta}=-\beta z+\Theta(x)$. The above symmetry imposes $\Theta(x)=-\Theta(-x)$. By time averaging Eq. 6, taking into account the $z$ independence of velocity and Reynolds stresses profiles:

$$
\partial_{x}\left(\frac{\bar{p}}{\rho}+\left\langle v_{x}^{\prime 2}\right\rangle\right)=-g_{x} \alpha(-\beta z+\Theta(x)) .
$$

We took into account the independence of the time average $\bar{v}_{x}^{\prime 2}$ on $z$, so $\bar{v}_{x}^{\prime 2}=\left\langle v_{x}^{\prime 2}\right\rangle$, and $\bar{v}_{x}=\left\langle v_{x}\right\rangle=0$.

Integrating in $x$, and differenciating in $z$ gives:

$$
g_{x} \alpha \beta x-\partial_{z} \frac{\bar{p}}{\rho}=\varphi(z)
$$

where $\varphi$ only depends on $z$, and appeared due to the integration on $x$..

By time averaging Eq. 7:

$$
\partial_{x}\left\langle v_{x}^{\prime} v_{z}^{\prime}\right\rangle=-\partial_{z} \frac{\bar{p}}{\rho}-g_{z} \alpha(-\beta z+\Theta(x))+\nu \partial_{x x}^{2}\left\langle v_{z}\right\rangle .
$$

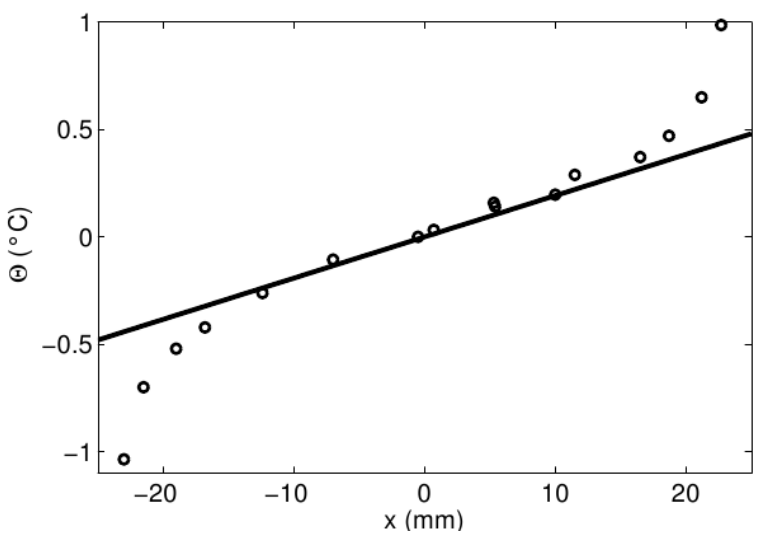

FIG. 5: Comparison between the measured temperature profile (circles) and the slope deduced from the measure of the transverse Reynolds stress, through equation 12 , where we neglect the term proportional to $\beta$. The applied power $P=62.7 \mathrm{~W}$ and $\psi=20^{\circ}$.

Separating the $x$ and $z$ dependent parts, we obtain:

$$
\begin{array}{r}
g_{z} \alpha \Theta(x)+g_{x} \alpha \beta x-\nu \partial_{x x}^{2}\left\langle v_{z}\right\rangle+\partial_{x}\left\langle v_{x}^{\prime} v_{z}^{\prime}\right\rangle \\
=\varphi(z)+g_{z} \alpha \beta z .
\end{array}
$$

As they depend on independent variables, quantities on both sides must be constant. This constant is zero, as the left hand side of the equation is an odd function of $x$. Thus:

$$
\partial_{x} \sigma=-g_{z} \alpha(\Theta(x)+\tan (\psi) \beta x),
$$

where we use the notation:

$$
\sigma=\left\langle v_{x}^{\prime} v_{z}^{\prime}\right\rangle-\nu \partial_{x}\left\langle v_{z}\right\rangle .
$$

In the following, we shall neglect the viscous term in $\sigma$. The ratio between $\left\langle v_{x}^{\prime} v_{z}^{\prime}\right\rangle$ and the viscous term is between 15 and 30 for the applied powers we consider here. This equation 12 relates $\Theta(x)$ and $\left\langle v_{x}^{\prime} v_{z}^{\prime}\right\rangle$, and constitutes our main access to $\Theta(x)$. However, we checked it by measuring the temperature profile, and comparing it with $-\partial_{x} \sigma / g_{z} \alpha$. For this comparison, shown in figure 5 , we approximated $\sigma$ with a quadratic function of $x$, and we neglected the $\operatorname{term} \tan (\psi) \beta x$, which is generally negligible, as remarked in [1].

Defining $Q_{x}=\left\langle v_{x} \theta\right\rangle$, and neglecting heat conduction, the energy conservation gives:

$$
\beta U_{z}=\partial_{x} Q_{x}
$$

This equation relates the transverse diffusive heat flux $Q_{x}$ and the longitudinal velocity $U_{z}$. It is our only access to $Q_{x}$, and thus to the effective transverse heat diffusivity.

These two equations 12 and 14 must be completed by two phenomenological equations [3]:

$$
\sigma \simeq\left\langle v_{x}^{\prime} v_{z}^{\prime}\right\rangle=-\nu_{t u r b} \partial_{x} U_{z}
$$


and:

$$
Q_{x}=-\kappa_{\text {turb }} \partial_{x} \Theta
$$

where $\nu_{\text {turb }}$ is the kinematic eddy viscosity, and $\kappa_{\text {turb }}$ is the eddy heat diffusivity.

The turbulent Prandtl number $P r_{\text {turb }}=\nu_{\text {turb }} / \kappa_{\text {turb }}$ will be a crucial quantity. We can estimate it in the following way. Let us assume a parabolic shape for $Q_{x}$, similar to the $\sigma$ one:

$$
Q_{x}=\frac{\beta d_{e f f}^{2}}{8}\left(\partial_{x} U_{z}(0)\right)\left(1-\frac{4 x^{2}}{d_{e f f}^{2}}\right),
$$

where we used equation 14. The turbulent Prandtl number is defined as:

$$
\begin{aligned}
P r_{\text {turb }} & =\frac{\sigma \partial_{x} \Theta}{Q_{x} \partial_{x} U_{z}} \\
& =\frac{16 U_{t}^{2} d^{2}}{-g_{z} \alpha \beta d_{\text {eff }}^{4}} \frac{\mathcal{C}_{\sigma}^{2}}{\mathcal{C}_{v}^{2}} \\
& =16 \frac{\mathcal{C}_{\sigma}^{2}}{\mathcal{C}_{v}^{2}} \frac{d^{4}}{d_{\text {eff }}^{4}}\left(\frac{Q_{z}}{\kappa \beta}\right)^{2 / 3}\left(\frac{\kappa^{2}}{-g_{z} \alpha \beta d^{4}}\right)^{1 / 3} \\
& =16 \frac{\mathcal{C}_{\sigma}^{2}}{\mathcal{C}_{v}^{2}} \frac{d^{4}}{d_{\text {eff }}^{4}}\left(\frac{N u^{2}}{R a P r}\right)^{1 / 3},
\end{aligned}
$$

where we used the definition of $U_{t}$ (equation 1 ), $\mathcal{C}_{v}$ (equation 4 ) and $\mathcal{C}_{\sigma}$ (equation 5).

$$
N u=\frac{Q_{z}}{\kappa \beta} \quad, \quad R a=\frac{-g_{z} \alpha \beta d^{4}}{\nu \kappa},
$$

are the Nusselt and the Rayleigh numbers, and $\operatorname{Pr}=$ $\nu / \kappa$, where $\kappa$ is the heat diffusivity. In this expression of $\operatorname{Pr}_{\text {turb }}, \mathcal{C}_{\sigma}, \mathcal{C}_{v}$, and $d_{\text {eff }}$ are constant for a given inclination angle $\psi$, as the renormalized profiles of the velocity and the Reynolds shear stress merge, both for Soft and Hard Turbulence. In the Hard Turbulence, $\Gamma=N u / \sqrt{R a P r}$ is also constant, so $P_{\text {turb }}$ should be constant. In the Soft Turbulence, however, $\Gamma$ varies rapidly, increasing with increasing $P$ (thus $U_{t}$ ).

In figure 6 , we show the turbulent Prandtl number versus $U_{t}$ for the three inclination angles $\psi=5^{\circ}, 10^{\circ}$, and $20^{\circ}$. The large uncertainty is consistent with the cumulated uncertainties on $\mathcal{C}_{\sigma}, \mathcal{C}_{v}$ and $\Gamma$. Within this uncertainty, $P r_{\text {turb }}$ can be considered as constant in the Hard Turbulence (full symbols). As expected, it varies rapidly in the Soft Turbulence (open symbols). However, it accidentally remains close to the Hard Turbulence value. Despite the large uncertainty, the data are consistent with a smooth tendency in the Hard Turbulence regime (full symbols), giving larger $P r_{\text {turb }}$ when the inclination angle is larger. Gibert et al. [8] found $P r_{\text {turb }} \simeq 0.4$ for the vertical case $\left(\psi=0^{\circ}\right)$. This tendency is in agreement with previous works $[5,18]$.

In the two next sections, V and VI, we examine the consequences of mixing length models for $\nu_{\text {turb }}$. The eddy heat diffusivity $\kappa_{\text {turb }}$ will be deduced from the experimental value of $\operatorname{Pr}_{t u r b}$.

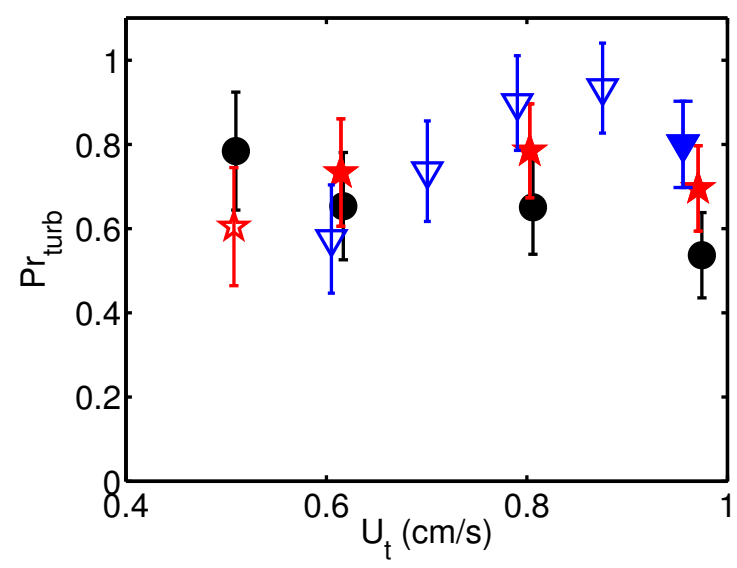

FIG. 6: The turbulent Prandtl number versus $U_{t}$ for the three inclination angles $\psi=5^{\circ}$ (circles), $\psi=10^{\circ}$ (stars), $\psi=20^{\circ}$ (triangles). Open symbols are for the Soft Turbulence regime, full symbols correspond to the Hard Turbulence one.

\section{MIXING LENGTH APPROXIMATION}

The eddy viscosity $\nu_{\text {turb }}$ can be seen as the product of a squared mixing length $\ell^{2}$, and a characteristic frequency $\omega_{o}$ :

$$
\nu_{\text {turb }}=\omega_{o} \ell^{2} .
$$

Without stratification, the only characteristic frequency is given by the velocity gradient:

$$
\omega_{o}=\left|\partial_{x} U_{z}\right|,
$$

which gives the following expression for the transverse Reynolds stress:

$$
\sigma=\left\langle v_{x}^{\prime} v_{z}^{\prime}\right\rangle=-\ell^{2}\left|\partial_{x} U_{z}\right| \partial_{x} U_{z} \quad,
$$

which corresponds to the well known mixing length $[2,3]$ approximation. Let us for a time take it as valid. In the same spirit, we shall take for the heat flux:

$$
Q_{x}=-\frac{\ell^{2}}{P r_{\text {turb }}}\left|\partial_{x} U_{z}\right| \partial_{x} \Theta
$$

where $P r_{\text {turb }}$ is the turbulent Prandtl number, we shall assume independent of coordinates.

In our flow, the transverse stress, which is also the momentum flux, is zero at the limit of the boundary layer, where its sign changes. Within the boundary layer, the momentum flux is oriented towards the wall. Out of the boundary layer, it is oriented towards the other side of the channel. As for the heat flux, it is zero at the walls. It is indeed almost zero at the limit of the boundary layer.

In order to obtain undimensional equations, we shall define $\sigma=U_{o}^{2} s, x=\ell \xi, U_{z}=U_{o} u, \Theta=\Theta_{o} \tau$, and $Q_{x}=Q_{o} q$, with:

$$
U_{o}^{2}=-g_{z} \alpha \Theta_{o} \ell, Q_{o}=U_{o} \beta \ell, \Theta_{o}=\beta \ell P r_{t u r b},
$$


The equations 12, 14, 15, 16 now become:

$$
\begin{gathered}
s^{\prime}=\tau \quad, \quad q^{\prime}=u, \\
s=-u^{\prime 2} \quad, \quad q=-u^{\prime} \tau^{\prime},
\end{gathered}
$$

valid as long as $u^{\prime}>0$. We note at this stage that all the $\psi$ dependent parameters have disappeared from the equations. Thus, we shall not be able to interpret the evolution of the velocity profile shape with the inclination angle $\psi$. However, equations 25 and 26 allow an analytical solution, which can act as a reference.

If $\xi$ is small, we can write $u=u^{\prime}(0) \xi$ et $\tau=\tau^{\prime}(0) \xi$. We then obtain $s=s(0)+\tau^{\prime}(0) \xi^{2} / 2$ et $q=q(0)+u^{\prime}(0) \xi^{2} / 2$, with $s(0)=-u^{\prime 2}(0)$ and $q(0)=-u^{\prime}(0) \tau^{\prime}(0)$. As $s$ and $q$ must vanish together at the boundary, it is tempting to try as boundary condition: $u^{\prime}(0)=\tau^{\prime}(0)$. It gives $u=\tau$ and $s=q$ everywhere.

Thanks to the hypothesis $\tau=u$, we can write $\left(u^{\prime}>0\right)$ :

$$
s^{\prime}=-2 u^{\prime} u^{\prime \prime}=u \text {. }
$$

Following a well known method, we define $u^{\prime}=\phi(u)$, which gives $u^{\prime \prime}=\phi \phi^{\prime}$, and:

$$
-2 \phi^{2} \phi^{\prime}=-\frac{2}{3}\left(\phi^{3}\right)^{\prime}=u, \phi^{3}=\phi_{o}^{3}-\frac{3}{4} u^{2} .
$$

We then have:

$$
\frac{d u}{d \xi}=\left(\frac{3}{4}\right)^{1 / 3}\left(u_{m}^{2}-u^{2}\right)^{1 / 3},
$$

and:

$$
\begin{aligned}
\xi & =\left(\frac{4}{3}\right)^{1 / 3} \int_{0}^{u} \frac{d v}{\left(u_{m}^{2}-v^{2}\right)^{1 / 3}} \\
& =\left(\frac{4 u_{m}}{3}\right)^{1 / 3} \int_{0}^{u / u_{m}} \frac{d v}{\left(1-v^{2}\right)^{1 / 3}} .
\end{aligned}
$$

We thus have an universal velocity profile shape, whose amplitude $u_{m}$ is related to the initial slope $u^{\prime}(0)$ by:

$$
u^{\prime}(0)=\left(\frac{3}{4}\right)^{1 / 3} u_{m}^{2 / 3}
$$

This shape is not very far, but different, from a sine shape. Indeed, if the exponent of the denominator in the integral, equation 30 , were $1 / 2$ instead of $1 / 3$, we should have a sine. But it simply would correspond to a constant $\nu_{\text {turb }}$, independent of $\partial_{x} U_{z}$. On the other hand, we are now able to evaluate the error made on $d \Theta / d x$ when approximating $\sigma$ with a quadratic function, as we made in previous papers $[1,9]$. It is equivalent to approximating $s$ with:

$$
s_{q}=s(0)\left(1-\frac{\xi^{2}}{\xi_{m}^{2}}\right)
$$

where

$$
\xi_{m}=\left(\frac{4 u_{m}}{3}\right)^{1 / 3} \int_{0}^{1} \frac{d v}{\left(1-v^{2}\right)^{1 / 3}} .
$$

We thus take $s_{q}^{\prime \prime}(0)=-2 s(0) / \xi_{m}^{2}$ as the slope of $\tau$, instead of $s^{\prime \prime}(0)=u^{\prime}(0)$. The ratio is:

$$
\begin{aligned}
K & =\frac{s_{q}^{\prime \prime}(0)}{s^{\prime \prime}(0)}=-\frac{2 s(0)}{u^{\prime}(0) \xi_{m}^{2}} \\
& =\frac{2(3 / 4)^{1 / 3} u_{m}^{2 / 3}}{\left(4 u_{m} / 3\right)^{2 / 3}\left(\int_{0}^{1} \frac{d v}{\left(1-v^{2}\right)^{1 / 3}}\right)^{2}} \simeq 0.896 .
\end{aligned}
$$

The error is thus about $10 \%$, which justifies this approximation for interpreting experimental data and evaluating $d \Theta / d x$, as we made in figure 5 .

To resume, while qualitatively interesting, the standard mixing length $[2,3]$ approximation is unable not only to reproduce the evolution of the experimental profile when $\psi$ is increased, but disagrees with the experimental profile for small $\psi$. Strikingly, for small $\psi$, the profile better corresponds to a constant $\nu_{\text {turb }}$.

\section{INFLUENCE OF STRATIFICATION}

\section{A. Correction to mixing length}

As remarked above, the standard mixing length $[2,3$, 5] approximation, equation 22, is unable to explain the evolution of the velocity profile shape as the inclination angle $\psi$ is increased. Indeed, this approximation does not take into account the stratification due to the transverse temperature gradient and the transverse component of the gravity. A fluid element goes upward or downward, vertically, depending if it is hotter or colder than the surrounding. It results in a correlation between axial $(z)$ and transverse $(x)$ components of the velocity, which has the opposite sign compared to the turbulent momentum transfer. But this physical mechanism seems unable to explain the failure of the equation 22 in the small $\psi$ case.

The competition between turbulence and stratification can be expressed in another way. Let us come back to the previous expression of the eddy viscosity:

$$
\nu_{t u r b}=\omega_{o} \ell^{2} .
$$

As noted before, without stratification, the characteristic frequency $\omega_{0}$ can only come from the velocity gradient:

$$
\omega_{o}=\left|\partial_{x} U_{z}\right|
$$

With a thermal stratification, however, a BruntVäisälä frequency $N$ [18] appears:

$$
N^{2}=-\alpha \vec{g} \cdot \operatorname{grad}(\bar{\theta}) .
$$


The competition between turbulence and stratification can be taken into account with the following expression for $\omega_{o}[16,17]$ :

$$
\begin{aligned}
\omega_{o} & =\left(\left(\partial_{x} U_{z}\right)^{2}+\epsilon^{\prime} \alpha \vec{g} \cdot \operatorname{grad}(\bar{\theta})\right)^{1 / 2} \\
& =\left(\left(\partial_{x} U_{z}\right)^{2}-\epsilon^{\prime} g_{z} \alpha \beta+\epsilon^{\prime} g_{x} \alpha \partial_{x} \Theta\right)^{1 / 2},
\end{aligned}
$$

where $\epsilon^{\prime}$ is an ajustable parameter. It gives:

$$
\sigma=-\ell^{2}\left(\left(\partial_{x} U_{z}\right)^{2}-\epsilon^{\prime} g_{z} \alpha \beta+\epsilon^{\prime} g_{x} \alpha \partial_{x} \Theta\right)^{1 / 2} \partial_{x} U_{z}
$$

In the same way, we shall take:

$$
Q_{x}=-\frac{\ell^{2}}{P r_{\text {turb }}}\left(\left(\partial_{x} U_{z}\right)^{2}-\epsilon^{\prime} g_{z} \alpha \beta+\epsilon^{\prime} g_{x} \alpha \partial_{x} \Theta\right)^{1 / 2} \partial_{x} \Theta
$$

Let us recall the two other equations:

$$
\partial_{x} \sigma=-g_{z} \alpha \Theta \quad, \quad \partial_{x} Q_{x}=\beta U_{z},
$$

where, again we neglect $\tan (\psi) \beta x$ compared to $\Theta$, as it is effectively negligible in our experiments. The two supplementary terms we introduce in $\omega_{o}$, equation 38 , have not the same role. The first one is positive, thus destabilizing, and increases the Reynolds stress. It does not depend on $x$. When it dominates on $\left(\partial_{x} U_{z}\right)^{2}$, it gives a constant turbulent viscosity, which can explain why, in the small $\psi$ case, the profile is close to a sine. The second term is stabilizing, and lowers the stress, thus damping the turbulence. It is $\psi$ dependent which can explain the evolution of the profile.

$\epsilon^{\prime}$ appears as an adjustable parameter. Note that the Richardson number, $R i$, can be defined as (gradient Richardson number [18]):

$$
R i=-\frac{\alpha \vec{g} \cdot \operatorname{grad}(\bar{\theta})}{\left(\partial_{x} U_{z}(0)\right)^{2}} .
$$

Then, $\epsilon^{\prime}$ appears as the inverse of a critical Richardson number, $R i_{c}=1 / \epsilon^{\prime}$. Indeed, for $R i>R i_{c}$, the frequency $\omega_{o}$ is not real. It then must be taken as zero, and both the Reynolds stress and the turbulent heat flux are zero: the flow is laminar.

In figure 7 we show all our measurements of the Richardson number within the Hard or Soft Turbulence regime. In the same figure, we show the critical Richardson number $R i_{c}$ for $\epsilon^{\prime}=20$, which will be justified in section VII.

\section{B. Non dimensional equations}

Equations 25 and 26 then become:

$$
\begin{gathered}
s^{\prime}=\tau \quad, \quad q^{\prime}=u, \\
s=-\varpi^{1 / 2} u^{\prime} \quad, \quad q=-\varpi^{1 / 2} \tau^{\prime},
\end{gathered}
$$

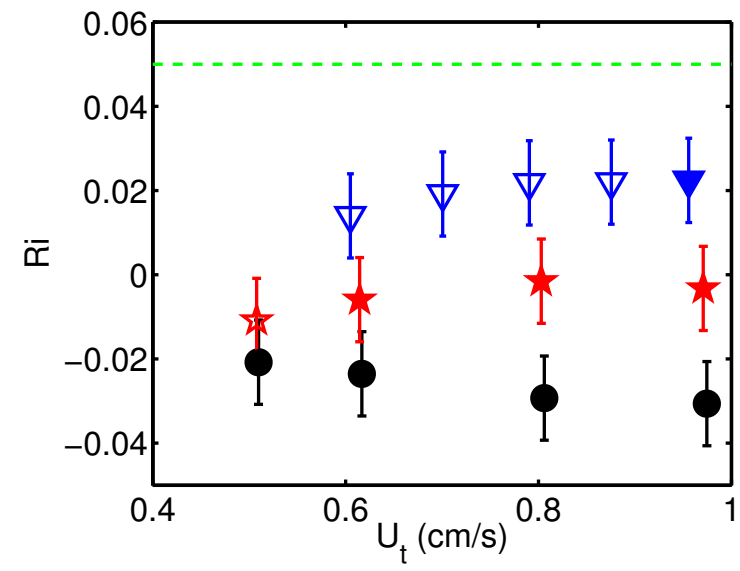

FIG. 7: The Richardson number within the Hard (full symbols) or Soft (open symbols) Turbulence regime, for $\psi=5^{\circ}$ (circles), $\psi=10^{\circ}$ (stars), and $\psi=20^{\circ}$ (triangles) versus $U_{t}$. The dashed line indicates $R i_{c}=1 / \epsilon^{\prime}$.

valid as long as $\varpi=u^{\prime 2}+\epsilon^{\prime} / P r_{\text {turb }}-\epsilon^{\prime} \tan (\psi) \tau^{\prime}>0$.

Again, there exists a solution where $u=\tau$ and $s=q$ everywhere, which makes $s$ and $q$ to vanish together at the boundary.

The shape of the velocity profile depends now on $\psi$ both through the $\tan (\psi)$ term and through the $\psi$ dependence of $P r_{\text {turb }}$. As previously (section V), we can estimate the range of variations of the quantity:

$$
K=-\frac{2 s(0)}{u^{\prime}(0) \xi_{m}^{2}} .
$$

As noted above, the velocity profile shape can go from a sine to a linear one. The linear profile corresponds to a quadratic one for $q$ (equation 43) thus for $s$ :

$$
u=u^{\prime}(0) \xi \Longrightarrow q=s=-\frac{\xi_{m}^{2} u^{\prime}(0)}{2}\left(1-\frac{\xi^{2}}{\xi_{m}^{2}}\right)
$$

and $K=1$. The sine profile corresponds to $q$ being a cosine:

$$
\begin{aligned}
u & =\frac{2 \xi_{m}}{\pi} u^{\prime}(0) \sin \left(\frac{\pi \xi}{2 \xi_{m}}\right) \\
& \Longrightarrow q=s=-u^{\prime}(0)\left(\frac{2 \xi_{m}}{\pi}\right)^{2} \cos \left(\frac{\pi \xi}{2 \xi_{m}}\right),
\end{aligned}
$$

and $K=8 / \pi^{2} \simeq 0.81$.

Thus, whatever the possible variations of $u^{\prime}(0), \xi_{m}, \epsilon^{\prime}$, and $P r_{\text {turb }}$, the quantity $K$ remains in a range of variations of $\pm 10 \%$. We shall use this property to estimate the value of $u^{\prime}(0)$.

\section{Evaluation of $u^{\prime}(0)$}

Indeed, an almost constant value for $K$ in equation 45 is not sufficient to determine $u^{\prime}(0)$. Although we can 


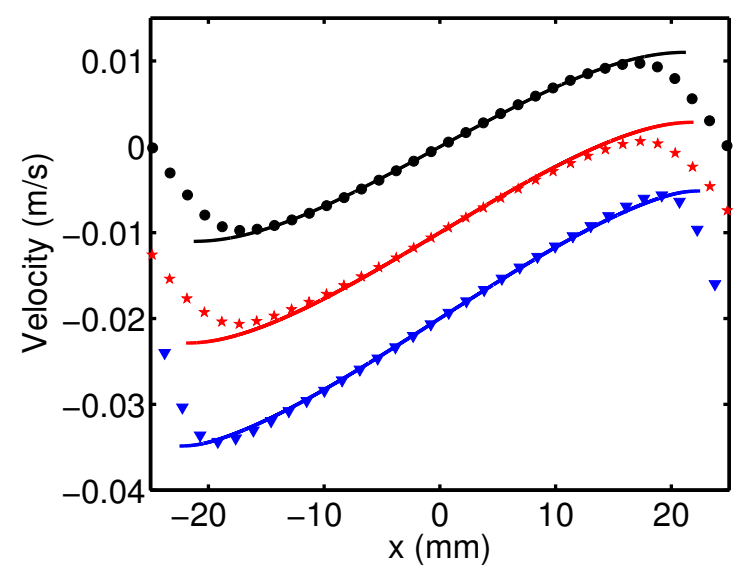

FIG. 8: Comparison of the theoretical profiles with the experimental ones. For determining the initial condition $u^{\prime}(0)$, we use equation 50, with $\epsilon^{\prime}=20$ and $A_{o}=74$. The characteristic velocity $U_{o}=\ell \sqrt{-g_{z} \alpha \beta P r_{\text {turb }}}$. The mixing length $\ell=d_{e f f} / 2 \xi_{m}$ and $\beta$ and $\operatorname{Pr}_{\text {turb }}$ are the experimental ones. The curves are shifted by $0.01 \mathrm{~m} / \mathrm{s}$ for clarity.

identify $\xi_{m}$ with the point where the Reynolds stress vanishes $x=d_{e f f} / 2$ :

$$
\xi_{m}=\frac{d_{e f f}}{2 \ell}
$$

the problem remains to evaluate $\ell$.

In fact, $\ell$ probably depends on the inclination angle $\psi$, but $\psi=0$ certainly corresponds to an extremum by symmetry $\psi \rightarrow-\psi$. As our range of $\psi$ is limited $\left(\psi \leq 20^{\circ}\right)$, considering $\ell$ as constant is a reasonable approximation. Then $\xi_{m}$ is also approximately constant, and the quasi constant value of $K$ allows to write:

$$
u^{\prime 2}(0)-\epsilon^{\prime} \tan (\psi) u^{\prime}(0)+\frac{\epsilon^{\prime}}{P r_{t u r b}}=A_{o}=\text { cste }
$$

As $u^{\prime}(0)$ must be positive:

$$
u^{\prime}(0)=\frac{\epsilon^{\prime} \tan (\psi)+\sqrt{\epsilon^{\prime 2} \tan (\psi)^{2}+4 A}}{2},
$$

where $A=A_{o}-\epsilon^{\prime} / P r_{\text {turb }}$.

Thus, we remain with two adjustable parameters for fiting all our velocity and Reynolds shear stress profiles, their shapes and their amplitudes, for every angle and applied power. We found that $\epsilon^{\prime}=20$ and $A_{o}=74$ were the best choice. They are correlated. The uncertainty on each one when the other is fixed, is small: of order 1 for $\epsilon^{\prime}$ and 2 for $A_{o}$. However, for $\epsilon^{\prime}$ above 25 or under 15, we found no agreement, whatever $A_{o}$ is. In the next section VII, we discuss the agreement between experiment and theory for this best choice.

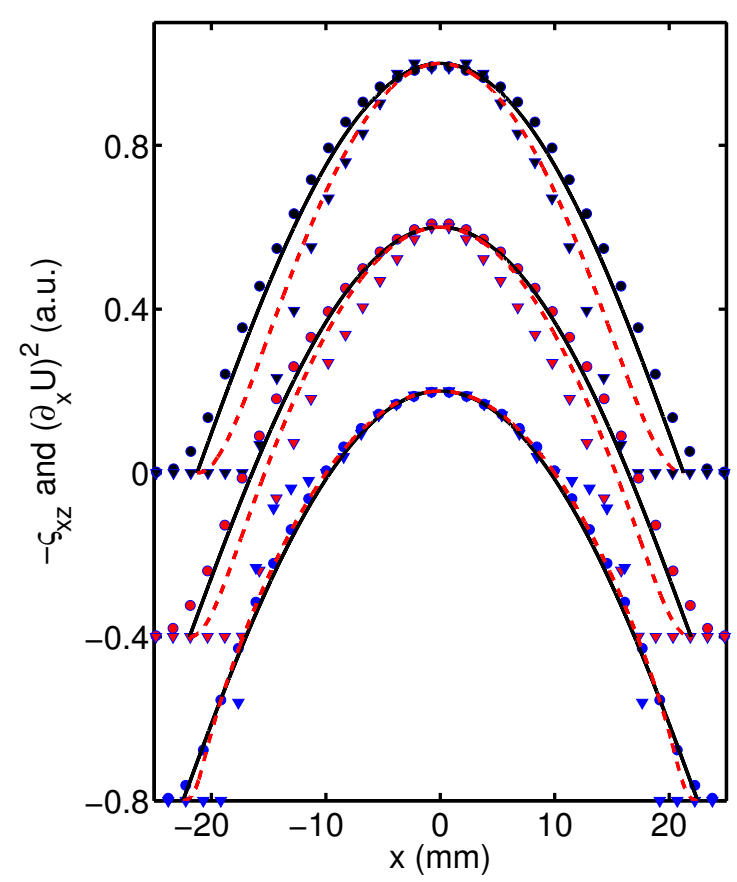

FIG. 9: Comparison of $-\sigma$ (circles and black continuous line) and $\left(\partial_{x} U_{z}\right)^{2}$ (triangles and red dashed line), both with their maximum normalized to 1 , in experiments (symbols) and theory (lines). The three groups are for $\psi=5^{\circ}$ (upper curves, black symbols), $\psi=10^{\circ}$ (middle curves, red symbols), and $\psi=20^{\circ}$ (lower curves, blue symbols). The groups are shifted by 0.4 for clarity.

\section{COMPARISON TO EXPERIMENTS AND DISCUSSION}

The figure 8 compares the experimental $U_{z}(x)$ and theoretical $U_{o} u(x / \ell)$ profiles for $\psi=5^{\circ}, 10^{\circ}$, and $20^{\circ}$ (applied power $P=77 \mathrm{~W}$ ). The normalizing velocity is $U_{o}=\ell \sqrt{-g_{z} \alpha \beta P r_{\text {turb }}}$ (equation 24). The mixing length $\ell$ is obtained from the identification $d_{e f f}=2 \xi_{m} \ell$, where $\xi_{m}$ is the non dimensional abscissa where $s$ vanishes. For $\beta$ we take the experimental value measured in [1], as well as for $P r_{\text {turb }}$ (see section IV). The initial condition $u^{\prime}(0)$ is given by the formula 50 with $\epsilon^{\prime}=20$ and $A_{o}=74$. The agreement can be considered as fair. Not only the slope at the origin is correct, but the profile shape has a similar evolution than the experimental one, being more and more linear as $\psi$ increases.

The $\psi$ dependence of $u^{\prime}(0)$ comes as well from the tan $\psi$ terms than from the $\psi$ dependence of $P r_{\text {turb }}$. Its amplitude is thus entirely governed by $\epsilon^{\prime}$, which is important to note, as the $\epsilon^{\prime}$ value obtained here is rather high, compared to other proposals [18]. A lower value would have made us unable to reproduce the $\psi$ dependence of $\partial_{x} U_{z}(0)$.

The high value of $\epsilon^{\prime}$ also reflects in the comparison of 
$-\sigma$ with $\left(\partial_{x} U_{z}\right)^{2}$. We show both quantities in figure 9, with their maximum value normalized to 1 . The experimental shape of $-\sigma$ is represented by circles and that of $\left(\partial_{x} U_{z}\right)^{2}$ by triangles. The theoretical profile of $-\sigma$ is shown as a continuous line and that of $\left(\partial_{x} U_{z}\right)^{2}$ by a dashed one. One can see that for $\psi=5^{\circ}$ (upper curves), the $\left(\partial_{x} U_{z}\right)^{2}$ profile is inside the $-\sigma$ one. For $\psi=20^{\circ}$ (lower curves), it is slightly outside. The theoretical curves qualitatively reproduce this evolution. Again, a large value of $\epsilon^{\prime}$ is necessary for this evolution.

This large value of $\epsilon^{\prime}$ is surprising as it implies a low value of the critical Richardson number $R i_{c}=1 / \epsilon^{\prime} \simeq$ 0.05 , above which the flow must be laminar. However, we can see in figure 7 that all our measured $R i$ in turbulent situation are smaller than $R i_{c}$. The Richardson number $R i$ increases when the inclination angle increases, and, for the higher inclination angles we explored, we found laminar or intermittent regimes. Comparing more precisely to previous works, we note that [19] found complete damping of turbulence for $R i=0.3$, and pointed some visible effect of stratification for $R i=0.05$. Znaien et al. [12] found intermittent or laminar regimes for $R i>0.05$ [20]. Odier et al. [5] observed a turbulent flow for $R i=0.08$, but their situation was more inhomogeneous than ours. Moreover, in all these experimental examples, account is only taken of the density gradient and gravity components transverse to the flow, not of the longitudinal components. This increases the value of $R i$. Thus, all these observations are in fair agreement with ours.

In our above comparison between experiment and theory, we choose the highest applied power. It thus corresponds to the Hard Turbulence regime. In this regime, $P r_{\text {turb }}$, thus $u^{\prime}(0)$ (equation 50), and the ratio $U_{o} / U_{t}$ (equation 57), only depend on $\psi$. This explains the perfect scaling of the velocity profile with $U_{t}$ [1]. In the Soft Turbulence regime, the rapid variation of $P r_{t u r b}$ with $P$ (or $U_{t}$, see figure 18), should influence both the shape of the velocity profile, and the value of $u^{\prime}(0)$. Thus, the extension of the above scaling to this regime, which lead us to assimilate Soft and Hard Turbulence [1], could be fortuitous.

We also can verify the hypothesis $\tau^{\prime}(0)=u^{\prime}(0)$ :

$$
\tau^{\prime}=s^{\prime \prime}=\ell^{2} \frac{\partial_{x x}^{2} \sigma}{U_{o}^{2}}
$$

and

$$
u^{\prime}=\ell \frac{\partial_{x} U_{z}}{U_{o}}
$$

thus:

$$
\frac{\tau^{\prime}(0)}{u^{\prime}(0)}=\frac{\ell}{U_{o}} \frac{\partial_{x x}^{2} \sigma}{\partial_{x} U_{z}}=\frac{4 \ell}{d_{e f f}} \frac{d}{d_{e f f}} \frac{\mathcal{C}_{\sigma}}{\mathcal{C}_{v}} \frac{U_{t}}{U_{o}} .
$$

We must compare the two characteristic velocities, $U_{t}$ and $U_{o}$ :

$$
\begin{aligned}
\frac{U_{t}^{3}}{U_{o}^{3}} & =\frac{-g_{z} \alpha Q_{z} d}{\left(-g_{z} \alpha \beta \ell^{2} P r_{\text {turb }}\right)^{3 / 2}} \\
& =\frac{1}{\operatorname{Pr}_{\text {turb }}^{3 / 2}} \frac{Q_{z}}{\kappa \beta}\left(\frac{\kappa^{2}}{-g_{z} \alpha \beta d^{4}}\right)^{1 / 2} \frac{d^{3}}{\ell^{3}}
\end{aligned}
$$

which gives:

$$
\frac{U_{t}^{3}}{U_{o}^{3}}=\frac{8 \xi_{m}^{3}}{P r_{t u r b}^{3 / 2}} \frac{d^{3}}{d_{e f f}^{3}} \frac{N u}{(R a P r)^{1 / 2}} .
$$

Coming back to equation 53:

$$
\frac{\tau^{\prime}(0)}{u^{\prime}(0)}=\frac{4}{P r_{\text {turb }}^{1 / 2}} \frac{d^{2}}{d_{\text {eff }}^{2}} \frac{\mathcal{C}_{\sigma}}{\mathcal{C}_{v}} \Gamma^{1 / 3},
$$

where $\Gamma=N u / \sqrt{R a P r}, N u$ is the Nusselt number, $R a$, the Rayleigh number (equations 19), and $\operatorname{Pr}=\nu / \kappa$, the Prandtl number. The value of the right hand side of equation 56 is 1 , using equation 18. All our hypothesis are thus consistent. Equation 53 allows to give a simpler expression for $U_{t} / U_{o}$ :

$$
\frac{U_{t}}{U_{o}}=\frac{d_{\text {eff }}^{2}}{4 d \ell} \frac{\mathcal{C}_{v}}{\mathcal{C}_{\sigma}}=\xi_{m}^{2} \frac{\ell}{d} \frac{\mathcal{C}_{v}}{\mathcal{C}_{\sigma}}
$$

It does not depend on $\Gamma$, which explains why it is constant as well in the Soft Turbulence regime.

We finally can give an estimate of the mixing length $\ell$. The value we obtain for $\xi_{m}$ is $\xi_{m} \simeq 4.4$. The effective width $d_{\text {eff }}$ is always close to $44 \mathrm{~mm}$. This gives $\ell=d_{e f f} / 2 \xi_{m} \simeq 5 \mathrm{~mm}$. It would seem natural that this value be affected close to the walls, when the distance to the wall is less than $\ell$. This could explain the poor agreement between experiment and theory in this range. However, our experimental technique (PIV) is not precise close to the walls, due to light reflexions.

\section{CONCLUSION}

The free convection flow in the square heat pipe we study here is a model flow which allowed us to study significant details, like the shape of the profiles, and a fine comparison between shear stress and velocity gradients. It shows, contrary to the conclusion of previous works [5-7], that the most simple mixing length formula 22 is unable to reproduce these features. Buoyancy, both its driving and stratifying parts, compete with velocity gradient in the expression of turbulent viscosity and heat diffusivity.

The experimentally observed evolution of the velocity profile toward a linear one when the inclination angle increases clearly shows that, in a stratified situation, the transverse stress $\sigma$ does not only depend on the velocity gradient. The reason can be traced to the correlation 
the stratification creates between $z$ and $x$ velocity fluctuations, which opposes to the "diffusive" correlation due to the velocity gradient. Reciprocally, observing a velocity profile closer to a sine than the one corresponding to equation 22 shows that the Reynolds transverse stress goes down less rapidly than $\left(\partial_{x} U_{z}\right)^{2}$ when going from the center to the walls. This is due to the driving role of the longitudinal temperature gradient. The formula proposed by Eidson [17] well takes this into account.

On the other hand, the parameter $\epsilon^{\prime}$ we find in the Eidson formula, has a value $\left(\epsilon^{\prime}=20\right)$ surprisingly large. Values close to 5 are more usual [17]. Correlatively, the critical value it gives for the critical Richardson number $\left(R i_{c}=0.05\right)$ is lower than expected [5]. However, it is confirmed by the observation that, in none of the turbulent flows we observed, the Richardson number was larger than $R i_{c}$. Larger $R i$ always resulted in a laminar flow. It is also the case for the experimental measurements we know, as far as we take all the components of the density gradient into account, not only the transverse flow ones.

A generally accepted approximation is that turbulent shear stress and turbulent heat transfer are related through a constant turbulent Prandtl number $P r_{\text {turb }}$.
Within this approximation, we could evaluate $P r_{\text {turb }}$, at least for the Hard Turbulence regime. Its evolution, going to larger values when the stratification increases, is qualitatively in agreement with previous studies [5]. The value obtained for small inclination angles $\psi$ is also in agreement with our study of the vertical case [8, 9], as well as the profile shape. This justifies the procedure we used in these papers, taking into account the reversals by rectifying the flow.

\section{Acknowledgments}

We acknowledge stimulating discussions with Laurent Chevillard. Thanks are also due to Y. Hallez, J.P. Hulin, J. Magnaudet, F. Moisy, D. Salin and J. Znaien for many useful discussions within the ANR project GIMIC. We thank region Rhone-Alpes for financial support (Cible 2011, $\mathrm{n}^{\circ}$ 2770) . The post-doctoral position of $\mathrm{X}$. Riedinger was supported by the ANR-07-BLANC-0181 project.
[1] X. Riedinger, J.-C. Tisserand, F. Seychelles, B. Castaing, and F. Chillà, "Heat transport regimes in an inclined channel " Phys. Fluids 25, 015117 (2013).

[2] L. Prandtl, "Bericht über Untersuchungen zur ausgebildeten Turbulenz", Zs.v angew. Math. Mech. 5, 136 (1925)

[3] U. Frisch, "Turbulence", Cambridge University Press, (1995).

[4] F. Chillà, J. Schumacher, "New perspectives in turbulent Rayleigh-Benard convection", Eur. Phys. J. E 3558 (2012).

[5] P. Odier, J. Chen, M. K. Rivera, R. E. Ecke "Fluid Mixing in Stratified Gravity Currents: The Prandtl Mixing Length", Phys. Rev. Lett. 102, 134504 (2009).

[6] P. Odier, J. Chen, R. E. Ecke "Understanding and modeling turbulent fluxes and entrainment in a gravity current", Physica D 241, 260 (2012).

[7] D. Krug, M. Holzner, B. Luthi, M. Wolf, W. Kinzelbach, A. Tsinober, "Experimental study of entrainment and interface dynamics in a gravity current", Experiments in Fluids, 54, 1530 (2013).

[8] Gibert, M. Pabiou, H. Tisserand, J.-C. Gertjerenken, B. Castaing, B. Chillà, F. "Heat Convection in a Vertical Channel: Plumes versus Turbulent Diffusion", Phys. Fluids, 21, 035109. (2009).

[9] J.C. Tisserand, M. Creyssels, M. Gibert, B. Castaing, F. Chillà, "Convection in a vertical channel", NJP 12, 075024 (2010).

[10] T. Seon, J.P. Hulin, D. Salin, B. Perrin, E.J. Hinch, "Laser-induced fluorescence measurements of buoyancy driven mixing in tilted tubes", Phys. Fluids, 18, 041701 (2006).

[11] J. Znaien, Y. Hallez, F. Moisy, J. Magnaudet, J.P. Hulin,
D. Salin, E.J. Hinch, "Experimental and numerical investigations of flow structure and momentum transport in a turbulent buoyancy-driven flow inside a tilted tube", Phys. Fluids, 21, 115102 (2009).

[12] J. Znaien, F. Moisy, J.P. Hulin, "Flow structure and momentum transport for buoyancy driven mixing flows in long tubes at different tilt angles" , Phys. Fluids, 23, 035105 (2011).

[13] E. Calzavarini, D. Lohse, F. Toschi, R. Tripiccione, "Rayleigh and Prandtl number scaling in the bulk of Rayleigh-Bénard turbulence", Phys. Fluids, 17, 055107. (2005).

[14] M.R. Cholemari, J.H. Arakeri, "Axially homogeneous, zero mean flow buoyancy-driven turbulence in a vertical pipe", J. Fluid Mech. , 621, 69-102 (2009).

[15] Smagorinsky J., "General circulation experiments with the primitive equations" Mon. Weather Rev. 91, 99 (1963).

[16] S.J. Kimmel, J.A. Domaradzki, "Large eddy simulations of Rayleigh-Bénard convection using subgrid scale estimation model", Phys. Fluids 12, 169 (2000).

[17] T.M. Eidson, "Numerical simulation of the turbulent Rayleigh-Bénard problem", J. Fluid Mech. 158, 245 (1985).

[18] K. Kundu et I.M. Cohen, "Fluids Mechanics", Academic Press, New York (2008).

[19] A. A. Townsend, "Turbulent flow in a stably stratified atmosphere" J. Fluid Mech. 3, 361 (1958).

[20] In their notations, $R i=\sin (\theta) \tilde{\beta} /(\partial\langle\tilde{u}\rangle / \partial \tilde{z})^{2}$. We estimated $R i$ for their sets $\left(T_{2}\right)$ and $\left(T_{3}\right)$ and found $R i \simeq$ 0.046 and $R i \simeq 0.039$. 